\title{
Log-logistic parameters estimation using moving extremes ranked set sampling design
}

\author{
HE Xiao-fang CHEN Wang-xue* YANG Rui
}

\begin{abstract}
In statistical parameter estimation problems, how well the parameters are estimated largely depends on the sampling design used. In the current paper, a modification of ranked set sampling (RSS) called moving extremes RSS (MERSS) is considered for the estimation of the scale and shape parameters for the log-logistic distribution. Several traditional estimators and ad hoc estimators will be studied under MERSS. The estimators under MERSS are compared to the corresponding ones under SRS. The simulation results show that the estimators under MERSS are significantly more efficient than the ones under SRS.
\end{abstract}

\section{$\S 1 \quad$ Introduction}

Cost effective sampling is a problem of major concern in some experiments especially when the measurement of the characteristic of interest is costly or painful or time consuming. The method of ranked set sampling (RSS) provides an effective way to achieve observational economy in terms of precision achieved per unit of sampling. Initially the concept of RSS was introduced by McIntyre (1952) as a process of increasing the precision of the sample mean as an estimator of population mean. Ranking can be performed based on expert judgment, visual inspection or any means that does not involve actually quantifying the observations. In RSS one first draws $n^{2}$ units at random from the population and partitions them into $n$ sets of $n$ units. The $n$ units in each set are ranked without making actual measurements. From the first set of $n$ units the unit ranked lowest is chosen for actual quantification. From the second set of $n$ units the unit

\footnotetext{
Received: 2019-1-12. Revised: 2019-09-10.

MR Subject Classification: 62D05.

Keywords: moving extremes ranked set sample, best linear unbiased estimator, maximum likelihood estimator.

Digital Object Identifier(DOI): https://doi.org/10.1007/s11766-021-3720-y.

Supported by the National Natural Science Foundation of China(11901236), Scientific Research Fund of Hunan Provincial Science and Technology Department(2019JJ50479), Scientific Research Fund of Hunan Provincial Education Department(18B322) and Fundamental Research Fund of Xiangxi Autonomous Prefecture(2018SF5026).

* Corresponding author.

(C)The Author(s) 2021, corrected publication June 2021.

The original version of this article was revised due to a retrospective Open Access order.
} 
ranked second lowest is measured. The process is continued until the unit ranked largest is measured from the $n$-th set of $n$ units. If a larger sample size is required then the procedure can be repeated $k$ times.

Takahasi and Wakimoto (1968) established a very important statistical foundation for the theory of RSS. Later estimation of parameters of various commonly used distributions has been carried out using RSS (for details see Stokes (1995), Al-Saleh et al. (2003), Chen et al. (2004), Al-Saleh et al. (2009), Abu-Dayyeh et al. (2013), Chen et al. (2017), Chen et al. (2018) and Qian et al. (2019)). However, ranking accuracy affects the efficiency of the estimator. When the set size is large, ranking error tends to occur. In order to reduce the error of ranking and keep optimality inherited in the original RSS procedure, Al-Odat et al. (2001) introduced the concept of varied set size RSS, which is coined here as moving extremes RSS (MERSS).

The procedure of MERSS is described as follows:

1. Select $n$ simple random samples of sizes $1,2,3, \cdots, n$, respectively.

2. Order the elements of each set by visual inspection or by some other cheap method, without actual measurement of the characteristic of interest.

3. Measure accurately the maximum ordered observation from the first set, then the second set, ..., the last set.

4. Step (3) is repeated on another $n$ sets of size $1,2,3, \cdots, n$, respectively, however the minimum ordered observations are measured instead of the maximum ordered observations.

5 . If needed, this process can be replicated $k$ times (cycles).

Clearly, only the two extreme values are used in MERSS, maximum or minimum of sets of varied size, whereas the ranks of all the elements of each set are needed in RSS. Since it is not difficult to identify maximum or minimum units, MERSS is a very useful modification of RSS. It allows for an increase of set size without introducing too many ranking errors.

AL-Saleh et al. (2003a) studied maximum likelihood estimator (MLE) of location parameter for normal distribution based on MERSS. AL-Saleh et al. (2003b) considered the MLE of the mean of exponential distribution using MERSS and they showed that MLE of MERSS is always performed better than simple random sampling (SRS) numerically. Abu-Dayyeh et al. (2009) studied the modified MLE of the mean of the exponential distribution under MERSS. AlHadhrami et al. (2012) studied the Bayes estimators of the population mean of the normal distribution using MERSS and investigated its properties. For further introduction of MERSS refer to Chen et al. (2013), Chen et al. (2016) and Chen et al. (2019).

A random variable $X$ is said to have a $\log$-logistic distribution with the scale parameter $\alpha$ and the shape parameter $\beta$ if its distribution function is given by

$$
F(x)=\frac{x^{\beta}}{x^{\beta}+\alpha^{\beta}},
$$

where $x>0, \alpha>0$ and $\beta>0$. The probability density function (pdf) corresponding to the distribution function in (1) is then given by

$$
f(x)=\frac{\beta \alpha^{\beta} x^{\beta-1}}{\left(x^{\beta}+\alpha^{\beta}\right)^{2}} .
$$

We write $L L D(\alpha, \beta)$ to denote the distribution as defined in (1). The applications of log-logistic 
distribution are well known in wealth or income (see Fisk (1961)), hydrology for modelling stream flow rates and precipitation (see Shoukri et al. (1988)) and engineer of survival analysis (see Ashkar et al. (2003)). For further details on the importance and applications of a loglogistic distribution one may refer to Bennett (1983), Ahmad et al. (1988), Robson et al. (1999) and Geskus (2001).

Parameter estimation problems for the log-logistic distribution have been discussed by many authors. Among recent literature, Balakrishnan et al. (1987) studied the best linear unbiased estimator (BLUE) of the scale parameter of a log-logistic distribution under SRS. Chen (2006) discussed about the interval estimation for the shape parameter of the log-logistic distribution under SRS. Lesitha et al. (2013) provided an unbiased estimator and BLUE of the scale parameter of a log-logistic distribution under RSS. Further, inference on the parameters of the log-logistic distribution has been studied by many authors using SRS including Tiku et al. (1992), Gupta et al. (1999), Kus et al. (2006), Abbas et al. (2016) and He et al. (2020).

In this paper, we consider several traditional estimators and ad hoc estimators of the scale and shape parameters $\alpha$ and $\beta$ from $L L D(\alpha, \beta)$ based on MERSS. In Sect. 2, we study an unbiased estimator or modified unbiased estimator and BLUE or modified BLUE of $\alpha$ and $\beta$ in case when one parameter is known and ad hoc estimators in case when both parameters are unknown. In Sect. 3, we consider the MLEs of the parameters of this distribution. The relative efficiencies of all estimators are simulated and the conclusions will be presented in Sect. 4.

\section{$\S 2 \quad$ Several types of estimators}

In this section, we deal with several types of estimators of $\alpha$ and $\beta$ of the $L L D(\alpha, \beta)$ under MERSS:

(i) An unbiased estimator and BLUE of $\alpha$ defined from $L L D(\alpha, \beta)$ in which $\beta$ is known,

(ii) A modified unbiased estimator and modified BLUE of $\beta$ when $\alpha$ is known and

(iii) Ad hoc estimators of $\alpha$ and $\beta$ when $\alpha$ and $\beta$ are both unknown.

2.1 Unbiased estimator and BLUE of $\alpha$ when $\beta$ is known

Let $\left\{x_{1}, x_{2}, x_{3}, \cdots, x_{2 n}\right\}$ be a simple random sample of size $2 n$ from (1) in which $\beta$ is known. Then the pdf of $\frac{x_{i}}{\alpha}$ is

Let

$$
f^{1}(x)=\frac{\beta x^{\beta-1}}{\left(1+x^{\beta}\right)^{2}} .
$$

Then we have

$$
E\left(\frac{x_{i}}{\alpha}\right)=\gamma
$$

Thus the BLUE of $\alpha$ under SRS is given by

$$
E\left(\frac{x_{i}}{\gamma}\right)=\alpha
$$

$$
\hat{\alpha}_{S R S, B L U E}=\frac{1}{2 n} \sum_{i=1}^{2 n} \frac{x_{i}}{\gamma} \text {. }
$$

Let $\left\{x_{11}, x_{22}, x_{33}, \cdots, x_{n n}, y_{11}, y_{12}, y_{13}, \cdots, y_{1 n}\right\}$ be a moving extremes ranked set sample 
of size $2 n$ from (1) in which $\beta$ is known. Then $\frac{x_{i i}}{\alpha}$ has the same density as the $i t h$ order statistic of an SRS of size $i$ from $f^{1}(x)(\operatorname{see} \operatorname{David}(1981))$, i.e. the pdf of $\frac{x_{i i}}{\alpha}$ is

$$
f_{i i}^{1}(x)=\frac{i \beta x^{i \beta-1}}{\left(1+x^{\beta}\right)^{i+1}} .
$$

Also $\frac{y_{1 i}}{\alpha}$ has the same density as the first order statistic of an SRS of size $i$ from $f^{1}(x)$ (see David(1981)), i.e. the pdf of $\frac{y_{1 i}}{\alpha}$ is

Let

$$
f_{1 i}^{1}(x)=\frac{i \beta x^{\beta-1}}{\left(1+x^{\beta}\right)^{i+1}} .
$$

with

$$
E\left(\frac{x_{i i}}{\alpha}\right)=\gamma_{i i}
$$

and

$$
\operatorname{Var}\left(\frac{x_{i i}}{\alpha}\right)=\sigma_{i i}^{2}
$$

with

$$
E\left(\frac{y_{1 i}}{\alpha}\right)=\gamma_{1 i}
$$

From (5)-(8), it can be seen that

$$
\operatorname{Var}\left(\frac{y_{1 i}}{\alpha}\right)=\sigma_{1 i}^{2} \text {. }
$$

$$
E\left(\frac{x_{i i}}{\gamma_{i i}}\right)=\alpha
$$

with

$$
\operatorname{Var}\left(\frac{x_{i i}}{\gamma_{i i}}\right)=\frac{\alpha^{2} \sigma_{i i}^{2}}{\gamma_{i i}^{2}}
$$

and

$$
E\left(\frac{y_{1 i}}{\gamma_{1 i}}\right)=\alpha
$$

with

$$
\operatorname{Var}\left(\frac{y_{1 i}}{\gamma_{1 i}}\right)=\frac{\alpha^{2} \sigma_{1 i}^{2}}{\gamma_{1 i}^{2}}
$$

Thus an UE of $\alpha$ under MERSS is given by

$$
\hat{\alpha}_{M E R S S, U E}=\frac{1}{2 n} \sum_{i=1}^{n}\left(\frac{x_{i i}}{\gamma_{i i}}+\frac{y_{1 i}}{\gamma_{1 i}}\right) .
$$

According to the lemma (see Casella et al., 2002, p.338), combine (9)-(11) with (12), we have

$$
\hat{\alpha}_{M E R S S, B L U E}=\frac{1}{2}\left(\sum_{i=1}^{n} \frac{\gamma_{1 i}^{2} \gamma_{i i}^{2}}{\gamma_{1 i}^{2} \sigma_{i i}^{2}+\gamma_{i i}^{2} \sigma_{1 i}^{2}}\right)^{-1} \sum_{i=1}^{n} \frac{\gamma_{1 i} \gamma_{i i}\left(x_{i i} \gamma_{1 i}+y_{1 i} \gamma_{i i}\right)}{\gamma_{1 i}^{2} \sigma_{i i}^{2}+\gamma_{i i}^{2} \sigma_{1 i}^{2}} .
$$

2.2 Modified unbiased estimator and modified BLUE of $\beta$ when $\alpha$ is known

Let $\left\{x_{1}, x_{2}, x_{3}, \cdots, x_{2 n}\right\}$ be a simple random sample of size $2 n$ from (1) in which $\alpha$ is known. Then the pdf of $\beta \ln \frac{x_{i}}{\alpha}$ is

$$
f^{2}(x)=\frac{e^{x}}{\left(1+e^{x}\right)^{2}} .
$$

Note that $E\left(\beta \ln \frac{x_{i}}{\alpha}\right)=0$. Hence we consider the estimators of $\beta$ based on order statistics $\beta \ln \frac{x_{(1)}}{\alpha} \leq \beta \ln \frac{x_{(2)}}{\alpha} \cdots \leq \beta \ln \frac{x_{(2 n)}}{\alpha}$.

Let

$$
E\left(\beta \ln \frac{x_{(i)}}{\alpha}\right)=\xi_{(i)},
$$


then it can be seen that

Thus the BLUE of $\frac{1}{\beta}$ under SRS is given by

$$
E\left(\frac{\ln \frac{x_{(i)}}{\alpha}}{\xi_{(i)}}\right)=\frac{1}{\beta} .
$$

$$
\frac{1}{2 n} \sum_{i=1}^{2 n} \frac{\ln \frac{x_{(i)}}{\alpha}}{\xi_{(i)}} .
$$

Then we suggest the following estimator of $\beta$

$$
\hat{\beta}_{S R S, M B L U E}=2 n\left(\sum_{i=1}^{2 n} \frac{\ln \frac{x_{(i)}}{\alpha}}{\xi_{(i)}}\right)^{-1},
$$

which will be called the modified unbiased estimator of $\beta$.

Let $\left\{x_{22}, x_{33}, x_{44}, \cdots, x_{n+1 n+1}, y_{12}, y_{13}, y_{14}, \cdots, y_{1 n+1}\right\}$ be a moving extremes ranked set sample of size $2 n$ from $L L D(\alpha, \beta)$ with $\alpha$ is known. Then $\beta \ln \frac{x_{i i}}{\alpha}$ has the same density as the $i t h$ order statistic of an SRS of size $i$ from $f^{2}(x)$, i.e. the pdf of $\beta \ln \frac{x_{i i}}{\alpha}$ is

$$
f_{i i}^{2}(x)=\frac{i e^{i x}}{\left(1+e^{x}\right)^{i+1}} .
$$

Also $\beta \ln \frac{y_{1 i}}{\alpha}$ has the same density as the first order statistic of an SRS of size $i$ from $f^{2}(x)$, i.e. the pdf of $\beta \ln \frac{y_{1 i}}{\alpha}$ is

Let

$$
f_{1 i}^{2}(x)=\frac{i e^{x}}{\left(1+e^{x}\right)^{i+1}} .
$$

with

$$
E\left(\beta \ln \frac{x_{i i}}{\alpha}\right)=\xi_{i i}
$$

and

$$
\operatorname{Var}\left(\beta \ln \frac{x_{i i}}{\alpha}\right)=\delta_{i i}^{2}
$$

with

$$
E\left(\beta \ln \frac{y_{1 i}}{\alpha}\right)=\xi_{1 i}
$$

Then we have

$$
\operatorname{Var}\left(\beta \ln \frac{y_{1 i}}{\alpha}\right)=\delta_{1 i}^{2},
$$

with

$$
E\left(\frac{\ln \frac{x_{i i}}{\alpha}}{\xi_{i i}}\right)=\frac{1}{\beta}
$$

and

$$
\operatorname{Var}\left(\frac{\ln \frac{x_{i i}}{\alpha}}{\xi_{i i}}\right)=\frac{\delta_{i i}^{2}}{\xi_{i i}^{2} \beta^{2}}
$$

with

$$
E\left(\frac{\ln \frac{y_{1 i}}{\alpha}}{\xi_{1 i}}\right)=\frac{1}{\beta}
$$

$$
\operatorname{Var}\left(\frac{\ln \frac{y_{1 i}}{\alpha}}{\xi_{1 i}}\right)=\frac{\delta_{1 i}^{2}}{\xi_{1 i}^{2} \beta^{2}} .
$$

Thus an unbiased estimator of $\frac{1}{\beta}$ under MERSS is given by

$$
\frac{1}{2 n} \sum_{i=2}^{n+1}\left(\frac{\ln \frac{y_{1 i}}{\alpha}}{\xi_{1 i}}+\frac{\ln \frac{x_{i i}}{\alpha}}{\xi_{i i}}\right) \text {. }
$$


Then we suggest the following estimator of $\beta$

$$
\hat{\beta}_{M E R S S, M U E}=2 n\left(\sum_{i=2}^{n+1} \frac{\ln \frac{y_{1 i}}{\alpha}}{\xi_{1 i}}+\frac{\ln \frac{x_{i i}}{\alpha}}{\xi_{i i}}\right)^{-1},
$$

which will be called the modified unbiased estimator of $\beta$. According to the lemma (see Casella et al., 2002, p.338), combine(21)-(23) with (24), we can obtain the BLUE of $\frac{1}{\beta}$ under MERSS

$$
-\frac{1}{2} \sum_{i=2}^{n+1} \frac{\left(\xi_{i i} \ln \frac{y_{1 i}}{\alpha}+\xi_{1 i} \ln \frac{x_{i i}}{\alpha}\right) \xi_{1 i} \xi_{i i}}{\xi_{1 i}^{2} \delta_{i i}^{2}+\xi_{i i}^{2} \delta_{1 i}^{2}}\left(\sum_{i=2}^{n+1} \frac{\xi_{1 i}^{2} \xi_{i i}^{2}}{\xi_{1 i}^{2} \delta_{i i}^{2}+\xi_{i i}^{2} \delta_{1 i}^{2}}\right)^{-1} .
$$

Then we suggest the following estimator of $\beta$

$$
\hat{\beta}_{M E R S S, M B L U E}=-2 \sum_{i=2}^{n+1} \frac{\xi_{1 i}^{2} \xi_{i i}^{2}}{\xi_{1 i}^{2} \delta_{i i}^{2}+\xi_{i i}^{2} \delta_{1 i}^{2}}\left[\sum_{i=2}^{n+1} \frac{\left(\xi_{i i} \ln \frac{y_{1 i}}{\alpha}+\xi_{1 i} \ln \frac{x_{i i}}{\alpha}\right) \xi_{1 i} \xi_{i i}}{\xi_{1 i}^{2} \delta_{i i}^{2}+\xi_{i i}^{2} \delta_{1 i}^{2}}\right]^{-1},
$$

which will be called the modified BLUE of $\beta$.

2.3 Ad hoc estimators of $\alpha$ and $\beta$

If $Z=\ln X$, then the pdf of $Z$

$$
f_{3}(z)=\frac{e^{-((z-\theta) / \lambda)}}{\lambda\left[1+e^{-((z-\theta) / \lambda)}\right]^{2}},
$$

where $\theta=\ln \alpha$ and $\lambda=\frac{1}{\beta}$.

Let $\left\{z_{1}, z_{2}, z_{3}, \cdots, z_{2 n}\right\}$ be a simple random sample of size $2 n$ from (27) and

$$
U_{(i)}=\frac{z_{(i)}-\theta}{\lambda}
$$

where $z_{(1)} \leq z_{(2)} \leq \cdots \leq z_{(2 n)}$. Denote $a_{i}=E\left(U_{(i)}\right)$ and $v_{i j}=\operatorname{cov}\left(U_{(i)}, U_{(j)}\right)$. The most well known estimators of location parameter $\theta$ and scale parameter $\lambda$ using the order statistics, are the BLUEs (Arnold et al., 1992 and Balakrishnan et al., 1992) which can be written as

$$
\hat{\theta}_{S R S}=-a^{\prime} C W
$$

and

$$
\begin{gathered}
\text { where } a=\left(\begin{array}{c}
a_{1} \\
\vdots \\
a_{2 n}
\end{array}\right), l_{2 n}=\left(\begin{array}{c}
1 \\
\vdots \\
1
\end{array}\right), V=\left(v_{i j}\right)_{2 n \times 2 n}, W=\left(\begin{array}{c}
\hat{\lambda}_{S R S}=l_{2 n}^{\prime} C W, \\
\vdots \\
z_{(2 n)}
\end{array}\right), \\
C=\frac{V^{-1}\left(l_{2 n} a^{\prime}-a l_{2 n}^{\prime}\right) V^{-1}}{d} \text { and } d=\left(l_{2 n}^{\prime} V^{-1} l_{2 n}\right)\left(a^{\prime} V^{-1} a\right)-\left(l_{2 n}^{\prime} V^{-1} a\right)^{2} .
\end{gathered}
$$

Then we suggest the following estimators of $\alpha$ and $\beta$

$$
\tilde{\alpha}_{S R S, M B L U E}=e^{-a^{\prime} C W}
$$

and

$$
\tilde{\beta}_{S R S, M B L U E}=\frac{1}{l_{2 n}^{\prime} C W}
$$

which will be called the modified BLUE of $\alpha$ and $\beta$, respectively.

Let $\left\{z_{11}, z_{22}, z_{33}, \cdots, z_{n n}, w_{11}, w_{12}, w_{13}, \cdots, w_{1 n}\right\}$ be a moving extremes ranked set sample of size $2 n$ from (27). Denote its order statistics as $z_{(1)}^{*} \leq z_{(2)}^{*} \leq \ldots \leq z_{(2 n)}^{*}$. The following ad hoc estimators of $\alpha$ and $\beta$ are the same as the estimators under SRS, expect that the statistics 
under SRS are replaced with their counterparts under MERSS. Denote

$$
U_{(i)}^{*}=\frac{z_{(i)}^{*}-\theta}{\lambda}
$$

$a_{i}^{*}=E\left(U_{(i)}^{*}\right)$ and $v_{i j}^{*}=\operatorname{cov}\left(U_{(i)}^{*}, U_{(j)}^{*}\right)$.

Then we can obtain ad hoc estimators of $\alpha$ and $\beta$

$$
\tilde{\alpha}_{M E R S S, A H E}=e^{-a^{*^{\prime}} C^{*} W^{*}}
$$

and

$\tilde{\beta}_{M E R S S, A H E}=\frac{1}{l_{2 n}^{\prime} C^{*} W^{*}}$,
where $a^{*}=\left(\begin{array}{c}a_{1}^{*} \\ \vdots \\ a_{2 n}^{*}\end{array}\right), V^{*}=\left(v_{i j}^{*}\right)_{2 n \times 2 n}, W^{*}=\left(\begin{array}{c}z_{(1)}^{*} \\ \vdots \\ z_{(2 n)}^{*} \\ 2\end{array}\right), C^{*}=\frac{V^{*-1}\left(l_{2 n} a^{*^{\prime}}-a^{*} l_{2 n}^{\prime}\right) V^{*-1}}{d}$ and $d^{*}=\left(l_{2 n}^{\prime} V^{*-1} l_{2 n}\right)\left(a^{*^{\prime}} V^{*-1} a^{*}\right)-\left(l_{2 n}^{\prime} V^{*-1} a^{*}\right)^{2}$.

\section{$\S 3 \quad$ MLEs}

In this section, we consider MLEs of the parameters of $L L D(\alpha, \beta)$ under MERSS. Under some regularity conditions, the asymptotic efficiency of the MLEs can be obtained from the inverse of the Fisher information matrix.

The fisher information matrix for $\alpha$ and $\beta$ under SRS

$$
I_{S R S}(\alpha, \beta)=\left(\begin{array}{cc}
I_{11} & I_{12} \\
I_{12} & I_{22}
\end{array}\right)=\left(\begin{array}{cc}
\frac{2 n \beta^{2}}{3 \alpha^{2}} & 0 \\
0 & \frac{2 n\left(3+\pi^{2}\right)}{9 \beta^{2}}
\end{array}\right)
$$

is given by Reath et al. (2018).

Let $\left\{x_{11}, x_{22}, x_{33}, \cdots, x_{n n}, y_{11}, y_{12}, y_{13}, \cdots, y_{1 n}\right\}$ be a moving extremes ranked set sample of size $2 n$ from $L L D(\alpha, \beta)$, then the pdfs of $x_{i i}$ and $y_{1 i}(i=1,2, \ldots, m)$ are respectively

and

$$
f_{i i}(x)=\frac{i \beta\left(\frac{x}{\alpha}\right)^{i \beta-1}}{\alpha\left[1+\left(\frac{x_{i i}}{\alpha}\right)^{\beta}\right]^{i+1}}
$$

$$
f_{1 i}(x)=\frac{i \beta\left(\frac{x}{\alpha}\right)^{\beta-1}}{\alpha\left[1+\left(\frac{x}{\alpha}\right)^{\beta}\right]^{i+1}} .
$$

In order to get the MLEs, we start with the likelihood function

$$
\begin{aligned}
L_{M E R S S}(\alpha, \beta) & =\prod_{i=1}^{n} f_{i i}\left(x_{i i}\right) f_{1 i}\left(y_{1 i}\right) \\
& =\prod_{i=1}^{n} \frac{i \beta\left(\frac{x_{i i}}{\alpha}\right)^{i \beta-1}}{\alpha\left[1+\left(\frac{x_{i i}}{\alpha}\right)^{\beta}\right]^{i+1}} \frac{i \beta\left(\frac{y_{1 i}}{\alpha}\right)^{\beta-1}}{\alpha\left[1+\left(\frac{y_{1 i}}{\alpha}\right)^{\beta}\right]^{i+1}} .
\end{aligned}
$$


The log-likelihood function is

$$
\begin{aligned}
\ln L_{M E R S S} & =C+2 n \ln \beta-2 n \ln \alpha+\sum_{i=1}^{n}(i \beta-1) \ln \frac{x_{i i}}{\alpha}+\sum_{i=1}^{n}(\beta-1) \ln \frac{y_{1 i}}{\alpha} \\
& -\sum_{i=1}^{n}(i+1) \ln \left[1+\left(\frac{x_{i i}}{\alpha}\right)^{\beta}\right]-\sum_{i=1}^{n}(i+1) \ln \left[1+\left(\frac{y_{1 i}}{\alpha}\right)^{\beta}\right],
\end{aligned}
$$

where $C$ is a constant. Then we have

$$
\frac{\partial \ln L_{M E R S S}}{\partial \alpha}=-\frac{\beta}{\alpha}\left[\frac{n(n+3)}{2}-\sum_{i=1}^{n} \frac{(i+1)\left(\frac{x_{i i}}{\alpha}\right)^{\beta}}{1+\left(\frac{x_{i i}}{\alpha}\right)^{\beta}}-\sum_{i=1}^{n} \frac{(i+1)\left(\frac{y_{1 i}}{\alpha}\right)^{\beta}}{1+\left(\frac{y_{1 i}}{\alpha}\right)^{\beta}}\right]
$$

and

$$
\frac{\partial \ln L_{M E R S S}}{\partial \beta}=\frac{2 n}{\beta}+\sum_{i=1}^{n} i \ln \frac{x_{i i}}{\alpha}+\sum_{i=1}^{n} \ln \frac{y_{1 i}}{\alpha}-\sum_{i=1}^{n} \frac{(i+1)\left(\frac{x_{i i}}{\alpha}\right)^{\beta} \ln \frac{x_{i i}}{\alpha}}{1+\left(\frac{x_{i i}}{\alpha}\right)^{\beta}}-\sum_{i=1}^{n} \frac{(i+1)\left(\frac{y_{1 i}}{\alpha}\right)^{\beta} \ln \frac{y_{1 i}}{\alpha}}{1+\left(\frac{y_{1 i}}{\alpha}\right)^{\beta}} .
$$

The second-order derivative of $\alpha$ and $\beta$ for the $\ln L_{M E R S S}$ are computed as

$$
\begin{aligned}
& \frac{\partial^{2} \ln L_{M E R S S}}{\partial \alpha^{2}}=-\frac{\beta}{\alpha^{2}}\left[\frac{n(n+3)}{2}-\sum_{i=1}^{n} \frac{(i+1)\left(\frac{x_{i i}}{\alpha}\right)^{\beta}}{1+\left(\frac{x_{i i}}{\alpha}\right)^{\beta}}-\sum_{i=1}^{n} \frac{(i+1)\left(\frac{y_{1 i}}{\alpha}\right)^{\beta}}{1+\left(\frac{y_{1 i}}{\alpha}\right)^{\beta}}\right] \\
&-\frac{\beta^{2}}{\alpha^{2}}\left[\sum_{i=1}^{n} \frac{(i+1)\left(\frac{x_{i i}}{\alpha}\right)^{\beta}}{\left(1+\left(\frac{x_{i i}}{\alpha}\right)^{\beta}\right)^{2}}+\sum_{i=1}^{n} \frac{(i+1)\left(\frac{y_{1 i}}{\alpha}\right)^{\beta}}{\left(1+\left(\frac{y_{1 i}}{\alpha}\right)^{\beta}\right)^{2}}\right], \\
& \frac{\partial^{2} L_{M E R S S}}{\partial \beta^{2}}=-\frac{2 n}{\beta^{2}}-\sum_{i=1}^{n} \frac{(i+1)\left(\frac{x_{i i}}{\alpha}\right)^{\beta}\left(\ln \frac{x_{i i}}{\alpha}\right)^{2}}{\left[1+\left(\frac{x_{i i}}{\alpha}\right)^{\beta}\right]^{2}}-\sum_{i=1}^{n} \frac{(i+1)\left(\frac{y_{1 i}}{\alpha}\right)^{\beta}\left(\ln \frac{y_{1 i}}{\alpha}\right)^{2}}{\left[1+\left(\frac{y_{1 i}}{\alpha}\right)^{\beta}\right]^{2}}
\end{aligned}
$$

and

$$
\begin{aligned}
\frac{\partial^{2} \ln L_{M E R S S}}{\partial \alpha \partial \beta} & =-\frac{n(n+3)}{2 \alpha}+\frac{1}{\alpha} \sum_{i=1}^{n} \frac{(i+1)\left(\frac{x_{i i}}{\alpha}\right)^{\beta}}{1+\left(\frac{x_{i i}}{\alpha}\right)^{\beta}}+\frac{1}{\alpha} \sum_{i=1}^{n} \frac{(i+1)\left(\frac{y_{1 i}}{\alpha}\right)^{\beta}}{1+\left(\frac{y_{1 i}}{\alpha}\right)^{\beta}} \\
& +\frac{\beta}{\alpha} \sum_{i=1}^{n} \frac{(i+1)\left(\frac{x_{i i}}{\alpha}\right)^{\beta} \ln \frac{x_{i i}}{\alpha}}{\left(1+\left(\frac{x_{i i}}{\alpha}\right)^{\beta}\right)^{2}}+\frac{\beta}{\alpha} \sum_{i=1}^{n} \frac{(i+1)\left(\frac{y_{1 i}}{\alpha}\right)^{\beta} \ln \frac{y_{1 i}}{\alpha}}{\left(1+\left(\frac{y_{1 i}}{\alpha}\right)^{\beta}\right)^{2}}
\end{aligned}
$$

respectively. Thus we have

$$
\begin{aligned}
I_{11}^{*} & =-E\left(\frac{\partial^{2} \ln L_{M E R S S}}{\partial \alpha^{2}}\right) \\
& =\frac{n(n+3) \beta}{2 \alpha^{2}}-\frac{\beta^{2}}{\alpha^{3}} \sum_{i=1}^{n} \int_{0}^{\infty} \frac{i(i+1)\left(\frac{x}{\alpha}\right)^{i \beta+\beta-1}}{\left[1+\left(\frac{x}{\alpha}\right)^{\beta}\right]^{i+2}} d x-\frac{\beta^{2}}{\alpha^{3}} \sum_{i=1}^{n} \int_{0}^{\infty} \frac{i(i+1)\left(\frac{x}{\alpha}\right)^{2 \beta-1}}{\left[1+\left(\frac{x}{\alpha}\right)^{\beta}\right]^{i+2}} d x \\
& +\frac{\beta^{3}}{\alpha^{3}} \sum_{i=1}^{n} \int_{0}^{\infty} \frac{i(i+1)\left(\frac{x}{\alpha}\right)^{i \beta+\beta-1}}{\left[1+\left(\frac{x}{\alpha}\right)^{\beta}\right]^{i+3}} d x+\frac{\beta^{3}}{\alpha^{3}} \sum_{i=1}^{n} \int_{0}^{\infty} \frac{i(i+1)\left(\frac{x}{\alpha}\right)^{2 \beta-1}}{\left[1+\left(\frac{x}{\alpha}\right)^{\beta}\right]^{i+3}} d x \\
& =\frac{n(n+3) \beta}{2 \alpha^{2}}-\frac{\beta}{\alpha^{2}} \sum_{i=1}^{n} \int_{0}^{\infty} \frac{i(i+1) t^{i}}{(1+t)^{i+2}} d t-\frac{\beta}{\alpha^{2}} \sum_{i=1}^{n} \int_{0}^{\infty} \frac{i(i+1) t}{(1+t)^{i+2}} d t \\
& +\frac{\beta^{2}}{\alpha^{2}} \sum_{i=1}^{n} \int_{0}^{\infty} \frac{i(i+1) t^{i}}{(1+t)^{i+3}} d t+\frac{\beta^{2}}{\alpha^{2}} \sum_{i=1}^{n} \int_{0}^{\infty} \frac{i(i+1) t}{(1+t)^{i+3}} d t
\end{aligned}
$$


and

$$
\begin{gathered}
=\frac{n(n+3) \beta}{2 \alpha^{2}}-\frac{n(n+1) \beta}{2 \alpha^{2}}-\frac{n \beta}{\alpha^{2}}+\frac{2 \beta^{2}}{\alpha^{2}} \sum_{i=1}^{n}\left(1-\frac{2}{i+2}\right) \\
=\frac{2 \beta^{2}}{\alpha^{2}} \sum_{i=1}^{n}\left(1-\frac{2}{i+2}\right), \\
I_{22}^{*}=E\left\{\frac{2 n}{\beta^{2}}+\sum_{i=1}^{n} \frac{(i+1)\left(\frac{x_{i i}}{\alpha}\right)^{\beta}\left(\ln \frac{x_{i i}}{\alpha}\right)^{2}}{\left[1+\left(\frac{x_{i i}}{\alpha}\right)^{\beta}\right]^{2}}+\sum_{i=1}^{n} \frac{(i+1)\left(\frac{y_{1 i}}{\alpha}\right)^{\beta}\left(\ln \frac{y_{1 i}}{\alpha}\right)^{2}}{\left[1+\left(\frac{y_{1 i}}{\alpha}\right)^{\beta}\right]^{2}}\right\} \\
=\frac{2 n}{\beta^{2}}+\frac{1}{\beta^{2}} E\left[\sum_{i=1}^{n} \frac{(i+1) h_{i i} l^{2} h_{i i}}{\left(1+h_{i i}\right)^{2}}+\sum_{i=1}^{n} \frac{(i+1) s_{1 i} l^{2} s_{1 i}}{\left(1+s_{1 i}\right)^{2}}\right]
\end{gathered}
$$

$$
\begin{aligned}
I_{12}^{*} & =-E\left[-\frac{n(n+3)}{2 \alpha}+\frac{1}{\alpha} \sum_{i=1}^{n} \frac{(i+1)\left(\frac{x_{i i}}{\alpha}\right)^{\beta}}{1+\left(\frac{x_{i i}}{\alpha}\right)^{\beta}}+\frac{1}{\alpha} \sum_{i=1}^{n} \frac{(i+1)\left(\frac{y_{1 i}}{\alpha}\right)^{\beta}}{1+\left(\frac{y_{1 i}}{\alpha}\right)^{\beta}}\right. \\
& +\frac{\beta}{\alpha} \sum_{i=1}^{n} \frac{(i+1)\left(\frac{x_{i i}}{\alpha}\right)^{\beta} \ln \frac{x_{i i}}{\alpha}}{\left(1+\left(\frac{x_{i i}}{\alpha}\right)^{\beta}\right)^{2}}+\frac{\beta}{\alpha} \sum_{i=1}^{n} \frac{\left.(i+1)\left(\frac{y_{1 i}}{\alpha}\right)^{\beta} \ln \frac{y_{1 i}}{\alpha}\right]}{\left.\left(1+\left(\frac{y_{1 i}}{\alpha}\right)^{\beta}\right)^{2}\right]} \\
& =\frac{n(n+3)}{2 \alpha}-\frac{n(n+1)}{2 \alpha}-\frac{n}{\alpha}-\frac{\beta}{\alpha} E\left[\sum_{i=1}^{n} \frac{(i+1)\left(\frac{x_{i i}}{\alpha}\right)^{\beta} \ln \frac{x_{i i}}{\alpha}}{\left(1+\left(\frac{x_{i i}}{\alpha}\right)^{\beta}\right)^{2}}+\sum_{i=1}^{n} \frac{(i+1)\left(\frac{y_{1 i}}{\alpha}\right)^{\beta} \ln \frac{y_{1 i}}{\alpha}}{\left(1+\left(\frac{y_{1 i}}{\alpha}\right)^{\beta}\right)^{2}}\right] \\
& =-\frac{1}{\alpha} E\left[\sum_{i=1}^{n} \frac{(i+1) h_{i i} \operatorname{lnh}_{i i}}{\left(1+h_{i i}\right)^{2}}+\sum_{i=1}^{n} \frac{\left.(i+1) s_{1 i} \ln s_{1 i}\right]}{\left(1+s_{1 i}\right)^{2}}\right]
\end{aligned}
$$

where $s_{1 i}$ is 1 th order statistics and $h_{i i}$ is maximum order statistics of a moving extremes ranked set sample from $\operatorname{LLD}(1,1)$. Combining (38), (39) with (40), we have

$$
I_{M E R S S}(\alpha, \beta)=\left(\begin{array}{cc}
I_{11}^{*} & I_{12}^{*} \\
I_{12}^{*} & I_{22}^{*}
\end{array}\right)
$$

\section{$\S 4$ Numerical comparison}

In this section, we will compare the relative efficiencies of the above estimators in Sect.2 and Sect.3.

The efficiency of $\hat{\alpha}_{M E R S S, U E}$ with respect to (w.r.t.) $\hat{\alpha}_{S R S, B L U E}$ is

$$
e f f^{1}=\frac{M S E\left(\hat{\alpha}_{S R S, B L U E}\right)}{M S E\left(\hat{\alpha}_{M E R S S, U E}\right)},
$$

where MSE is an abbreviation of the mean square error. Similarly, denote $\hat{\alpha}_{M E R S S, M B L U E}$ w.r.t. $\hat{\alpha}_{S R S, B L U E}, \hat{\beta}_{M E R S S, M U E}$ w.r.t. $\hat{\beta}_{S R S, M B L U E}, \hat{\beta}_{M E R S S, M B L U E}$ w.r.t. $\hat{\beta}_{S R S, M B L U E}$, $\tilde{\alpha}_{M E R S S, A H E}$ w.r.t. $\tilde{\alpha}_{S R S, M B L U E}$ and $\tilde{\beta}_{M E R S S, A H E}$ w.r.t. $\tilde{\beta}_{S R S, M B L U E}$ as eff $f^{2}$, eff $f^{3}$, ef $f^{4}$, eff $f^{5}$ and $e f f^{6}$, respectively. It can be seen that $e f f^{i}(\mathrm{i}=3,4)$ are free of $\alpha$.

The asymptotic efficiencies $\hat{\alpha}_{M E R S S, M L E}$ w.r.t. $\hat{\alpha}_{S R S, M L E}, \hat{\beta}_{M E R S S, M L E}$ w.r.t. $\hat{\beta}_{S R S, M L E}$ 
and $\left(\hat{\alpha}_{M E R S S, M L E}, \hat{\beta}_{M E R S S, M L E}\right)$ w.r.t. $\left(\hat{\alpha}_{S R S, M L E}, \hat{\beta}_{S R S, M L E}\right)$ are respectively

$$
\begin{gathered}
\text { aef } f^{7}=\frac{I_{11}^{*}}{I_{11}}=\frac{3}{n} \sum_{i=1}^{n}\left(1-\frac{2}{i+2}\right) \\
\text { aeff } f^{8}=\frac{I_{22}^{*}}{I_{22}}=\frac{9}{2 n\left(3+\pi^{2}\right)}\left[2 n+E\left(\sum_{i=1}^{n} \frac{(i+1) h_{i i} l^{2} h_{i i}}{\left(1+h_{i i}\right)^{2}}+\sum_{i=1}^{n} \frac{(i+1) s_{1 i} l n^{2} s_{1 i}}{\left(1+s_{1 i}\right)^{2}}\right)\right]
\end{gathered}
$$

and

$$
\text { aeff } f^{9}=\frac{\left|I_{M E R S S}(\alpha, \beta)\right|}{\left|I_{S R S}(\alpha, \beta)\right|} \text {. }
$$

It can be seen that aef $f^{i}(\mathrm{i}=7,8,9)$ are free of $\alpha$ and $\beta$ and aef $f^{7}>1$ for $n>2$.

From Tables 1-4, we conclude the following:

(1) $e f f^{1}>1$, which means $\hat{\alpha}_{M E R S S, U E}$ is more efficient $\hat{\alpha}_{S R S, B L U E}$.

(2) $e f f^{2}>1$, which means $\hat{\alpha}_{M E R S S, B L U E}$ is more efficient $\hat{\alpha}_{S R S, B L U E}$.

(3) Comparing eff $f^{1}$ with $e f f^{2}$, we conclude that $\hat{\alpha}_{M E R S S, B L U E}$ is more efficient than $\hat{\alpha}_{M E R S S, U E}$.

(4) $e f f^{3}>1$, which means $\hat{\beta}_{M E R S S, M U E}$ is more efficient $\hat{\beta}_{S R S, M B L U E}$.

(5) $e f f^{4}>1$, which means $\hat{\beta}_{M E R S S, M B L U E}$ is more efficient $\hat{\beta}_{S R S, M B L U E}$.

(6) Comparing eff $f^{3}$ with $e f f^{4}$, we conclude that $\hat{\beta}_{M E R S S, M B L U E}$ is more efficient than $\hat{\beta}_{M E R S S, M U E}$.

(7) $e f f^{5}>1$, which means $\tilde{\alpha}_{M E R S S, A H E}$ is more efficient $\tilde{\alpha}_{M E R S S, M B L U E}$.

(8) $e f f^{6}>1$, which means $\tilde{\beta}_{M E R S S, A H E}$ is more efficient $\tilde{\beta}_{M E R S S, M B L U E}$.

(9) In conclusion, the estimators of $\alpha$ and $\beta$ under MERSS are more efficient than that of $\alpha$ and $\beta$ under SRS in Sect.2.

From Tables 5-7, we conclude the following:

(10) aef $f^{i}(i=7,8,9)>1$ and they are increase as $n$ increase.

(11) aef $f^{7}>1$, which means the MLE of $\alpha$ under MERSS is more efficient than the MLE of $\alpha$ under SRS.

(12) aef $f^{8}>1$, which means the MLE of $\beta$ under MERSS is more efficient than the MLE of $\beta$ under SRS.

(13) aef $f^{9}>1$, which means the MLEs of $\alpha$ and $\beta$ under MERSS is more efficient than the MLE of $\alpha$ and $\beta$ under SRS.

(14) In conclusion, the MLEs of $\alpha$ and $\beta$ under MERSS are more efficient than that of $\alpha$ and $\beta$ under SRS in Sect.3.

(15) In conclusion, the MERSS is more efficient than SRS in estimating the scale and shape parameters of the log-logistic distribution. 
Table 1. The efficiency of $\hat{\alpha}_{M E R S S, U E}$ w.r.t. $\hat{\alpha}_{S R S, B L U E}$

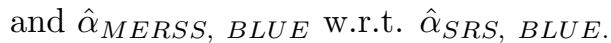

\begin{tabular}{llll}
\multicolumn{5}{c}{ and $\hat{\alpha}_{M E R S S, B L U E}$ w.r.t. $\hat{\alpha}_{S R S, B L U E .}$} \\
\hline$(\alpha, \beta)$ & $n$ & eff $f^{1}$ & eff $f^{2}$ \\
\hline$(1,3)$ & 6 & 1.71345 & 1.84017 \\
& 7 & 1.75349 & 1.93052 \\
$(2,3)$ & 8 & 1.65878 & 1.75611 \\
& 6 & 1.82032 & 1.84635 \\
& 7 & 1.53121 & 1.63641 \\
$(3,2)$ & 6 & 1.70422 & 1.74200 \\
& 7 & 4.08173 & 4.91487 \\
& 8 & 2.66697 & 2.72753 \\
\end{tabular}

Table 2. The efficiency of $\hat{\beta}_{M E R S S, M U E}$ w.r.t. $\hat{\beta}_{S R S, M B L U E}$ and $\hat{\beta}_{M E R S S, M B L U E}$ w.r.t. $\hat{\beta}_{S R S, M B L U E}$.

\begin{tabular}{clll}
\hline$\beta$ & $n$ & eff & eff \\
\hline 3 & 6 & 1.18180 & 2.91918 \\
& 7 & 1.61235 & 3.65413 \\
& 8 & 1.97523 & 3.91049 \\
4 & 6 & 1.14583 & 2.78112 \\
& 7 & 1.62455 & 3.25586 \\
5 & 8 & 1.90814 & 3.66518 \\
& 6 & 1.20770 & 2.83044 \\
& 7 & 1.46545 & 3.18749 \\
& 8 & 1.84847 & 3.56819 \\
\hline
\end{tabular}

Table 3. The efficiency of $\tilde{\alpha}_{M E R S S}, A H E$ w.r.t. $\tilde{\alpha}_{S R S, M B L U E}$.

\begin{tabular}{lll}
\hline$(\alpha, \beta)$ & $n$ & eff \\
\hline$(1,3)$ & 6 & 1.68089 \\
& 7 & 1.73293 \\
$(3,2)$ & 8 & 1.95351 \\
& 6 & 7.73763 \\
& 7 & 8.79398 \\
$(3,3)$ & 8 & 11.44288 \\
& 6 & 3.08259 \\
& 7 & 2.56829 \\
\hline
\end{tabular}


Table 4. The efficiency of $\tilde{\beta}_{M E R S S, A H E}$ w.r.t. $\tilde{\beta}_{S R S, M B L U E \text {. }}$

\begin{tabular}{lll}
\hline$(\alpha, \beta)$ & $n$ & eff \\
\hline$(1,3)$ & 6 & 1.27842 \\
& 7 & 1.68150 \\
$(3,2)$ & 8 & 1.83427 \\
& 6 & 3.08428 \\
& 7 & 3.64138 \\
$(3,3)$ & 8 & 2.94402 \\
& 6 & 4.55372 \\
& 7 & 2.90449 \\
& 8 & 3.53873 \\
\hline
\end{tabular}

Table 5. The asymptotic efficiency $\hat{\alpha}_{M E R S S, M L E}$ w.r.t. $\hat{\alpha}_{S R S, M L E}$.

\begin{tabular}{llll}
\hline$n$ & $\frac{\alpha^{2}}{\beta^{2}} I_{11}$ & $\frac{\alpha^{2}}{\beta^{2}} I_{11}^{*}$ & aeff \\
\hline 2 & 1.3333 & 1.6667 & 1.25000 \\
3 & 2.0000 & 2.8667 & 1.43335 \\
4 & 2.6667 & 4.2000 & 1.57498 \\
5 & 3.3333 & 5.6286 & 1.68855 \\
6 & 4.0000 & 7.1286 & 1.78215 \\
7 & 4.6667 & 8.6841 & 1.86087 \\
8 & 5.3333 & 10.2841 & 1.92828 \\
9 & 6.0000 & 11.9205 & 1.98675 \\
\hline
\end{tabular}

Table 6. The asymptotic efficiency $\hat{\beta}_{M E R S S, M L E}$ w.r.t. $\hat{\beta}_{S R S, M L E \text {. }}$

\begin{tabular}{llll}
\hline$n$ & $\beta^{2} I_{22}$ & $\beta^{2} I_{22}^{*}$ & aeff \\
\hline 2 & 5.7198 & 6.1550 & 1.07608 \\
3 & 8.5797 & 10.1005 & 1.17725 \\
4 & 11.4396 & 14.8545 & 1.29851 \\
5 & 14.2996 & 20.3281 & 1.42187 \\
6 & 17.1595 & 26.7176 & 1.55702 \\
7 & 20.0194 & 33.8705 & 1.69183 \\
8 & 22.8793 & 41.7207 & 1.82351 \\
9 & 25.7392 & 50.5267 & 1.96302 \\
\hline
\end{tabular}




Table 7 The asymptotic efficiency $\left(\hat{\alpha}_{M E R S S, M L E}, \hat{\beta}_{M E R S S, M L E}\right)$
\begin{tabular}{llll}
\multicolumn{4}{c}{ w.r.t. $\left(\hat{\alpha}_{S R S, M L E}, \hat{\beta}_{S R S, M L E .}\right)$} \\
\hline$n$ & $\alpha^{2}\left|I_{S R S}(\alpha, \beta)\right|$ & $\alpha^{2}\left|I_{M E R S S}(\alpha, \beta)\right|$ & aeff ${ }^{9}$ \\
\hline 2 & 7.6264 & 10.2586 & 1.34514 \\
3 & 17.1595 & 28.9966 & 1.68982 \\
4 & 30.5057 & 62.1831 & 2.03841 \\
5 & 47.6652 & 114.5466 & 2.40315 \\
6 & 68.6378 & 190.3164 & 2.77276 \\
7 & 93.4237 & 294.2395 & 3.14951 \\
8 & 122.0229 & 429.8509 & 3.52271 \\
9 & 154.4352 & 601.5848 & 3.89539 \\
\hline
\end{tabular}

Open Access This article is licensed under a Creative Commons Attribution 4.0 International License, which permits use, sharing, adaptation, distribution and reproduction in any medium or format, as long as you give appropriate credit to the original author(s) and the source, provide a link to the Creative Commons licence, and indicate if changes were made. The images or other third party material in this article are included in the articles Creative Commons licence, unless indicated otherwise in a credit line to the material. If material is not included in the articles Creative Commons licence and your intended use is not permitted by statutory regulation or exceeds the permitted use, you will need to obtain permission directly from the copyright holder. To view a copy of this licence, visit http://creativecomm ons.org/licenses/by/4.0/.

\section{References}

[1] K Abbas, Y C Tang. Objective bayesian analysis for log-logistic distribution, Communications in Statistics-Simulation and Computation, 2016, 45(8): 2782-2791.

[2] W Abu-Dayyeh, A Assrhani, K Ibrahim. Estimation of the shape and scale parameters of pareto distribution using ranked set sampling, Statistical Papers, 2013, 54(1): 207-225.

[3] W Abu-Dayyeh, E A Sawi. Modified inference about the mean of the exponential distribution using moving extreme ranked set sampling, Statistical Papers, 2009, 50(2): 249-259.

[4] M I Ahmad, C D Sinclair, A Werritty. Log-logistic flood frequency analysis, Journal of Hydrology, 1988, 98(3): 205-224.

[5] M T Al-Odat, M F Al-Saleh. A variation of ranked set sampling, Journal of Applied Statistical Science, 2001, 10(2): 137-146. 
[6] S A Al-Hadhrami, A I Al-Omari. Bayes Estimation of the Mean of Normal Distribution Using Moving Extreme Ranked Set Sampling, Pakistan Journal of Statistics and Operation Research, 2012, 8(1): 97-103.

[7] M F Al-Saleh, S A Al-Hadhrami. Parametric estimation for the location parameter for symmetric distributions using moving extremes ranked set sampling with application to trees data, Environmetrics, 2003a, 14(3): 651-664.

[8] M F Al-Saleh, S A Al-Hadhrami. Estimation of the mean of the exponential distribution using moving extremes ranked set sampling, Statistical Papers, 2003b, 44(3): 367-382.

[9] M F Al-Saleh, Y A Diab. Estimation of the parameters of Downton's bivariate exponential distribution using ranked set sampling scheme, Journal of Statistical Planning and Inference, 2009, 139(2): 277-286.

[10] B C Arnold, N Balakrishnan, H N Nagaraja. A first course in order statistics, Siam, 1992.

[11] F Ashkar, S Mahdi. Comparison of two fitting methods for the Log-logistic distribution, Water Resources Research, 2003, 39: 12-17.

[12] N Balakrishnan, A C Cohen. Order Statistics and Inference Estimation Methods, Biometrics, 1992, 48(2)

[13] N Balakrishnan, H J Malik. Best linear unbiased estimation of location and scale parameters of the log-logistic distribution, Communications in Statistics-Theory and Methods, 1987, 16(12): 3477-3495.

[14] S Bennett. Log-logistic regression models for survival data, Journal of the Royal Statistical Society, 1983, 32(2): 165-171.

[15] G Casella, R L Berger. Statistical inference, Pacific Grove, CA: Duxbury, 2002.

[16] W X Chen, Y Tian, M Y Xie. The global minimum variance unbiased estimator of the parameter for a truncated parameter family under the optimal ranked set sampling, Journal of Statistical Computation and Simulation, 2018, 88(17): 3399-3414.

[17] W X Chen, M Y Xie, M Wu. Parametric estimation for the scale parameter for scale distributions using moving extremes ranked set sampling, Statistics and Probability Letters, 2013, 83(9): 20602066.

[18] W X Chen, M Y Xie, M Wu. Modified maximum likelihood estimator of scale parameter using moving extremes ranked set sampling, Communications in Statistics Simulation and Computation, 2016, 45(6): 2232-2240.

[19] W X Chen, M Y Xie, M Wu. Maximum likelihood estimator of the parameter for a continuous one parameter exponential family under the optimal ranked set sampling, Journal of Systems Science and Complexity, 2017, 30(6): 1350-1363.

[20] W X Chen, R Yang, D S Yao, C X Long. Pareto parameters estimation using moving extremes ranked set sampling, Statistical Papers, 2019, https://doi.org/10.1007/s00362-019-01132-9

[21] Z H Chen, Z D Bai, B K Sinha. Ranked set sampling, theory and applications, Lecture Notes in Statistics, Springer, New York, 2004.

[22] Z M Chen. Estimating the shape parameter of the log-logistic distribution, International Journal of Reliability, Quality and Safety Engineering, 2006, 13(3): 257-266. 
[23] H A David. Order statistics (2edn), Wiley series in probability and statistics, New York, 1981.

[24] P R Fisk. The graduation of income distributions, Econometrica, 1961, 29(2): 171-185.

[25] R B Geskus. Methods for estimating the AIDS incubation time distribution when data of seroconversion is censored, Statistics in Medicine, 2001, 20: 795-812.

[26] R C Gupta, O Akman, S Lvin. A study of log-logistic model in survival analysis, Biometrical Journal, 1999, 41(4): 431-443.

[27] X F He, W X Chen, W S Qian. Maximum likelihood estimators of the parameters of the loglogistic distribution, Statistical Papers, 2020, 61(5): 1875-1892.

[28] C S Kus, M F Kaya. Estimation of parameters of the log-logistic distribution based on progressive censoring using the EM algorithm, Hacettepe Journal of Mathematics and Statistics, 2006, 35(2): 203-211.

[29] G Lesitha, P Y Thomas. Estimation of the scale parameter of a log-logistic distribution, Metrika, 2013, 76(3): 427-448.

[30] G A McIntyre. A method of unbiased selective sampling, using ranked sets, Australian Journal of Agricultural Research, 1952, 3(4): 385-390.

[31] W S Qian, W X Chen, X F He. Parameter estimation for the Pareto distribution based on ranked set sampling, Statistical Papers, 2019, https://doi.org/10.1007/s00362-019-01102-1.

[32] J Reath, J Dong, M Wang. Improved parameter estimation of the log-logistic distribution with applications, Computational Statistics, 2018, 33(1): 339-356.

[33] A Robson, D Reed. Statistical procedures for flood frequency estimation, Flood estimation handbook, Institute of Hydrology, Wallingford, p3, 1999.

[34] M M Shoukri, I U M Mian, D Tracy. Sampling properties of estimators of log-logistic distribution with application to Canadian precipitation data, Canadian Journal of Statistics, 1988, 16(3): 223-236.

[35] L Stokes. Parametric ranked set sampling, Annals of the Institute of Statistical Mathematics, 1995, 47(3): 465-482.

[36] K Takahasi, K Wakimoto. On unbiased estimates of the population mean based on the sample stratified by means of ordering, Annals of the Institute of Statistical Mathematics, 1968, 20(1): 1-31.

[37] M L Tiku, R P Suresh. A new method of estimation for location and scale parameters, Journal of Statistical Planning and Inference, 1992, 30: 281-292.

Department of Mathematics and Statistics, Jishou University, Jishou 416000, China.

Email: chenwangxue2017@163.com 